\title{
Author Correction: Avocado: a multi-scale deep tensor factorization method learns a latent representation of the human epigenome
}

\author{
Jacob Schreiber ${ }^{1}$, Timothy Durham², Jeffrey Bilmes ${ }^{1,3}$ and William Stafford Noble 1,2*
}

The original article can be found online at https://doi.org/10.1186/ s13059-020-01977-6.

* Correspondence: wnoble@uw.edu ${ }^{1}$ Paul G. Allen School of Computer Science and Engineering, University of Washington, Seattle, USA 2Department of Genome Sciences, University of Washington, Seattle, USA

Full list of author information is available at the end of the article
Correction to: Genome Biol 21, 81 (2020)

https://doi.org/10.1186/s13059-020-01977-6

Following publication of the original article [1], the following error was identified in the Results section, under the heading Avocado imputes epigenomic tracks more accurately than prior methods.

"Conversely, ChromImpute performs the best on MSE1obs (Avocado/ChromImpute $\mathrm{p}$ value $=2.37 \mathrm{e}-22$, PREDICTD/ChromImpute $\mathrm{p}$ value $=2.85 \mathrm{e}-12$ ) but the worst on MSE1imp, suggesting that it may over-call peaks. Additionally, Ernst and Kellis proposed six other evaluation performance measures, which show similar trends as the MSE1imp metric (Additional file 2: Fig. S2)."

The second sentence above should read (with the changed text in bold)

"Additionally, Ernst and Kellis proposed six other evaluation performance measures, which show similar trends as the MSE1obs metric (Additional file 2:

Fig. S2)."

Because this was a typo, the primary results and conclusions in the paper still hold. However, to improve the clarity of our claims in light of this correction, we would like to make two additional edits:

In the Background section, we would like to change:

"Using data from the Roadmap Epigenomics Consortium, we demonstrate that Avocado yields imputed values that are more accurate than those produced by

(c) The Author(s). 2021 Open Access This article is licensed under a Creative Commons Attribution 4.0 International License, which permits use, sharing, adaptation, distribution and reproduction in any medium or format, as long as you give appropriate credit to the original author(s) and the source, provide a link to the Creative Commons licence, and indicate if changes were made. The images or other third party material in this article are included in the article's Creative Commons licence, unless indicated otherwise in a credit line to the material. If material is not included in the article's Creative Commons licence and your intended use is not permitted by statutory regulation or exceeds the permitted use, you will need to obtain permission directly from the copyright holder. To view a copy of this licence, visit http://creativecommons.org/licenses/by/4.0/. The Creative Commons Public Domain Dedication waiver (http://creativecommons.org/publicdomain/zero/1.0/) applies to the data made available in this article, unless otherwise stated in a credit line to the data. 
either ChromImpute [6] or PREDICTD [5], as measured by multiple performance measures."

to

"Using data from the Roadmap Epigenomics Consortium, we demonstrate that Avocado yields imputed values that are more accurate than those produced by either ChromImpute [6] or PREDICTD [5], as measured by multiple performance measures based on MSE."

In the Conclusion section, we would like to change:

"This latent representation is trained to impute genome-wide epigenomics experiments, and we find that the resulting model outperforms prior methods at that task."

to

"This latent representation is trained to impute genome-wide epigenomics experiments, and we find that the resulting model outperforms prior methods at that task according to several performance measures based on MSE."

The original article [1] has been corrected.

Author details

${ }^{1}$ Paul G. Allen School of Computer Science and Engineering, University of Washington, Seattle, USA. ${ }^{2}$ Department of Genome Sciences, University of Washington, Seattle, USA. ${ }^{3}$ Department of Electrical Engineering, University of Washington, Seattle, USA.

Published online: 03 September 2021

Reference

1. Schreiber J, Durham T, Bilmes J, Noble WS. Avocado: a multi-scale deep tensor factorization method learns a latent representation of the human epigenome. Genome Biol. 2020;21:81 https://doi.org/10.1186/s13059-020-01977-6.

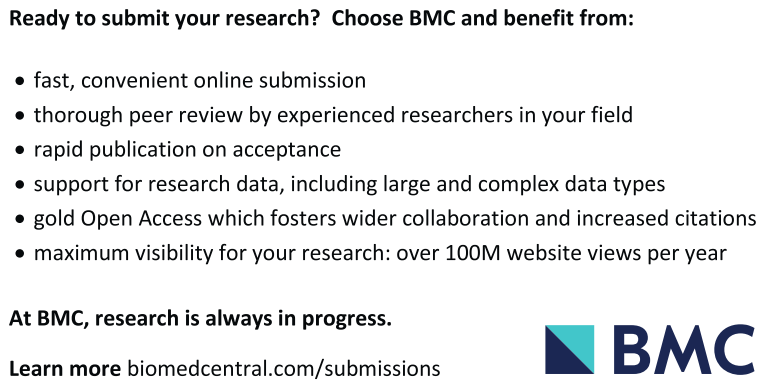

